\title{
ASSOCIAÇÃO ENTRE PERIODONTITE E BAIXO PESO AO NASCER: ESTUDO RETROSPECTIVO REALIZADO EM PUÉRPERAS DE 2005 A 2015 NO MUNICÍPIO DE FEIRA DE SANTANA
}

\author{
$\underline{\text { Ananda Lobo Pedreira Costa }}$; Isaac Suzart Gomes Filho²; Soraya Castro Trindade ${ }^{3}$ \\ 1. Bolsista PIBIC/CNPq, Graduanda em Odontologia, Universidade Estadual de Feira de Santana, e-mail: \\ anandalobo@yahoo.com.br \\ 2. Orientador, Departamento de Saúde, Universidade Estadual de Feira de Santana, e-mail: isuzart@gmail.com \\ 3. Participante do projeto, Departamento de Saúde, Universidade Estadual de Feira de Santana, e-mail: \\ soraya.castrotrindade@gmail.com
}

\section{PALAVRAS-CHAVE: PERIODONTITE; BAIXO PESO AO NASCER; PUÉRPERAS}

\section{INTRODUÇÃO}

As doenças periodontais representam um dos principais problemas de saúde bucal em todo o mundo (JIN et al., 2011). Consistem em um processo inflamatório no tecido periodontal, resultante do acúmulo de biofilme bacteriano na superfície dentária, sendo a sua ocorrência associada a condições socioeconômicas desfavoráveis, comportamentos prejudiciais à saúde como tabagismo, alcoolismo, dieta insatisfatória, higiene bucal deficiente e dificuldade de acesso aos serviços de saúde (GROSSI et al., 1995; VAN DYKE; VAN WINKELHOFF, 2013)

Nos últimos anos, foram realizados estudos que investigaram a possível correlação entre a presença de doenças periodontais e alterações sistêmicas, como desordens cardiovasculares, diabetes mellitus, osteoporose, infecções no trato respiratório, parto prematuro e baixo peso ao nascer, o que propiciou o surgimento de uma nova área de pesquisa comumente chamada de Medicina Periodontal ou Periodontia Médica (BRUNETTI; MORAES; MORAIS, 2007; SEYMOUR et al., 2007).

Ainda que as alterações periodontais sejam bastante prevalentes na população, elas podem ser observadas com maior frequência em algumas situações, como no período gestacional. O grande aumento dos níveis hormonais na gravidez ocasiona mudanças fisiológicas importantes no organismo da mulher, inclusive afetando o periodonto e levando à exacerbação ou ao estabelecimento de gengivites ou periodontites (GÜNCÜ; TÖZÜM; ÇAGLAYAN, 2005) A gestante torna-se mais sensível a algumas infecções, como as do trato genitourinário, que estão associadas a um aumento no risco de complicações gestacionais (HILLIER et al., 1995; MILANEZ, 2005).

Diante do exposto, o presente trabalho pretende avaliar a associação entre periodontite e baixo peso ao nascer em puérperas avaliadas em um período de dez anos no município de Feira de Santana.

\section{MATERIAL E MÉTODOS OU METODOLOGIA}

Para a presente investigação, será realizada extração dos dados originais dos bancos de pesquisa do NUPPIIM, alimentados com informações dos três períodos de estudo (2005 a 2015), referentes à condição socioeconômica-demográfica, história gestacional, hábitos de vida, aspectos relacionados com a saúde bucal, além dos dados relativos ao exame da cavidade bucal, que serão utilizados para avaliar a condição bucal das puérperas, através do diagnóstico da doença periodontal. Em seguida, será gerado um novo banco, unificando os dados provenientes de cada estudo, para a execução das análises. 
O tamanho da amostra do presente estudo será o somatório das amostras dos três estudos desenvolvidos entre os anos de 2005 a 2015. No primeiro estudo de intervenção, concluído em 2007, participaram 339 puérperas, enquanto que, para o segundo de caso-controle, concluído em 2012, foram avaliadas 371 puérperas e o terceiro estudo, também de casocontrole, em andamento, participaram até o momento 511 puérperas. $\mathrm{O}$ tamanho final da amostra será de 1.221 participantes.

\section{Definição das Variáveis:}

* Variável Independente: As gestantes/puérperas serão classificadas de acordo com os critérios da periodontite segundo Gomes Filho et al. (2007) e Page; Eke (2007) modificado por Eke et al. (2012).

* Variável dependente: Baixo Peso ao nascer. Será considerada a presença ou ausência do peso ao nascer <2500 gramas, de acordo com os critérios preconizados pela OMS.

* Covariáveis: serão consideradas as características sociodemográficas, de estilo de vida, história gestacional e condições pré-existentes.

Análise dos Dados

Neste trabalho, utilizando o banco de dados, será feita uma análise descritiva das variáveis, obtendo-se as frequências simples para as variáveis dicotômicas e medidas de tendência central e dispersão para as variáveis contínuas, de acordo com os grupos estudados.

\section{RESULTADOS E/OU DISCUSSÃO}

Ao final do período de coleta dos dados, 909 puérperas foram avaliadas. Destas, $14,30 \%$ (130) foram definidas com diagnóstico de periodontite e 85,70\% (779) sem este acometimento. A caracterização da amostra do estudo está apresentada nas tabelas 1 a 3.

As variáveis que apresentaram significância estatística entre os grupos foram: idade entre 18-35 anos $(\mathrm{p}<0,01)$, raça não branca $(\mathrm{p}=0,03)$, hipertensão $(\mathrm{p}=0,01)$, baixo peso ao nascer anterior $(\mathrm{p}<0,01)$ e visita ao dentista durante a gestação $(\mathrm{p}=0,05)$.

O grupo de puérperas com bebês de baixo peso apresentou a maior proporção para as covariáveis: baixo peso ao nascer anterior $(21,32 \%$ vs $9,41 \%)$ e hipertensão $(20,00 \%$ vs $12,96 \%$ ) quando comparado ao grupo de mães de bebês nascidos com peso superior a $2500 \mathrm{~g}$. Entretanto, apresentou em proporção menor, a idade $(67,39 \%$ vs $80,12 \%)$, raça não branca $(82,61 \%$ vs $90,57 \%)$ e visita ao dentista durante a gestação $(27,39 \%$ vs $34,46 \%)$.

A medida de associação entre periodontite e baixo peso ao nascer está apresentada na Tabela 3. De acordo com a razão de chances encontrada, OR = 2,63 (IC: 1,79 - 3,9), houve associação entre os eventos estudados.

De acordo com os achados do presente estudo, a periodontite apresentou associação com baixo peso ao nascer em puérperas. Tais achados corroboram com outros estudos que investigaram essa associação (Jeffcoat et al., 2001; Lopez et al., 2006; Saddik et al., 2008, Gomes -Filho et al., 2009). Entre alguns estudos que não demostraram associação, pode-se destacar uma coorte com 410 participantes (Michalowick et al. 2009) e um outro estudo casocontrole com 227 participantes (Rajapakse et al. 2008).

Os estudos que encontraram achados positivos para justificar a possibilidade de que a periodontite tenha um efeito sobre o baixo peso ao nascer está suportada na ação de determinados patógenos orais oportunistas e/ou a produtos liberados por estes, os quais, por via hematogênica podem desencadear o parto prematuro. Os mecanismos envolvidos nessa suposta associação ainda não são bem conhecidos (Lopez et al., 2006; Saddik et al., 2008) 
Tabela 1: Distribuição das características socioeconômico-demográficas e condição geral de saúde entre casos e controles- N = 909. Feira de Santana, Bahia - Brasil, 2016.

\begin{tabular}{|c|c|c|c|c|c|}
\hline \multicolumn{6}{|l|}{ Características } \\
\hline & \multirow{2}{*}{\multicolumn{2}{|c|}{$\begin{array}{l}\text { Grupo Controle } \\
\qquad N=679\end{array}$}} & \multirow{2}{*}{\multicolumn{2}{|c|}{$\begin{array}{c}\text { Grupo } \\
\text { Caso N = }\end{array}$}} & \multirow[b]{3}{*}{$\mathbf{P}$ * } \\
\hline & & & & & \\
\hline & $\mathbf{N}$ & $\%$ & & & \\
\hline \multicolumn{6}{|l|}{ Idade (em anos) } \\
\hline $18-35$ & 544 & 80,12 & 155 & 67,39 & $<0,01$ \\
\hline $14-17$ e $>35$ & 135 & 19,88 & 75 & 32,61 & \\
\hline \multicolumn{6}{|c|}{ Raça/Cor da pele materna } \\
\hline Branca & 64 & 9,43 & 34 & 13,48 & 0,03 \\
\hline Não branca & 615 & 90,57 & 196 & 82,61 & \\
\hline \multicolumn{6}{|l|}{$\begin{array}{l}\text { Baixo Peso ao nascer } \\
\text { anterior ** }\end{array}$} \\
\hline Sim & 43 & 9,41 & 29 & 21,32 & $<0,01$ \\
\hline Não & 414 & 90,59 & 107 & 78,68 & \\
\hline \multicolumn{6}{|l|}{ Hipertensão } \\
\hline Sim & 88 & 12,96 & 46 & 20,00 & 0,01 \\
\hline Não & 591 & 87,04 & 184 & 80,00 & \\
\hline
\end{tabular}

* Nível de significância estatística: $p \leq 0,05$;

Tabela 2: Distribuição dos hábitos relacionados à saúde entre casos (mães de bebês nascidos com peso $<2.500 \mathrm{~g}$ ) e controles (mães de bebês com peso $\geq 2.500 \mathrm{~g}$ ao nascimento) $-\mathrm{N}=909$. Feira de Santana, Bahia - Brasil, 2016.

\begin{tabular}{|c|c|c|c|c|c|c|}
\hline \multicolumn{7}{|c|}{ Visita ao dentista durante a gestação } \\
\hline \multirow{2}{*}{\multicolumn{3}{|c|}{$\begin{array}{l}\text { Grupo Controle } \\
\qquad N=679\end{array}$}} & \multirow{2}{*}{\multicolumn{3}{|c|}{$\begin{array}{c}\text { Grupo Caso } \\
\mathbf{N}=\mathbf{2 3 0}\end{array}$}} & \multirow[b]{3}{*}{$\mathbf{P}$ * } \\
\hline & & & & & & \\
\hline & $\mathbf{N}$ & $\%$ & & & $\%$ & \\
\hline Sim & & 234 & 34,46 & 63 & 27,39 & 0,05 \\
\hline Não & & 445 & 65,54 & 167 & 72,61 & \\
\hline
\end{tabular}

* Nível de significância estatística: $p \leq 0,05$;

Tabela 3: Associação entre a periodontite materna e o nascimento de bebês com peso inferior a 2500g. Feira de Santana, Bahia-Brasil-2016.

\begin{tabular}{l|l}
\hline OR & IC 95\% \\
\hline$(2,63)$ & $(1,79 \%-3,9 \%)$ \\
\hline
\end{tabular}

\footnotetext{
* Nível de significância estatística: $p \leq 0,05$;
} 


\section{CONSIDERAÇÕES FINAIS}

Uma associação entre a periodontite e o baixo peso ao nascer foi observada, entretanto os dados encontrados na literatura indicam a necessidade de estudos mais amplos, comparando os critérios adotados no diagnóstico da periodontite, já que a comparação dos trabalhos encontrados revela a fragilidade no diagnóstico da mesma, dificultando a comparabilidade e discussão dos seus achados, não sendo possível a conclusão categórica de que existe ou não a influência da periodontite no baixo peso ao nascer. Dessa maneira, o presente estudo reforça a necessidade de mais pesquisas, com critérios robustos para esclarecer essa questão que ainda apresenta lacunas do conhecimento.

\section{REFERÊNCIAS}

BRUNETTI, M. C.; MORAES, R. G. B.; MORAIS, T. M. N. Periodontia médica - uma mudança de paradigma na Odontologia. Fundamentos São Paulo: Artes Médicas, 2007.

CRUZ, S. S. et al. Doença periodontal materna como fator associado ao baixo peso ao nascer. Revista de Saúde Pública, São Paulo, v. 39, n. 5, p. 782-787, 2005.

GOMES-FILHO, I. S. et al. Exposure measurement in the association between periodontal disease and prematurity/low birth weight. Journal of Clinical Periodontology, Copenhagen, v. 34, n.11, p. 957-63, nov. 2007.

GOMES-FILHO, I. S. et al. Avaliação prospectiva da periodontite materna e baixo peso ao nascer. Revista Periodontia, v. 19, n. 4, p. 121-128, 2009.

GROSSI, G.; GENCO, E. E.; MACHTEI, A. W. Assessment of risk for periodontal disease. II. Risk indicators for alveolar bone loss. Journal of Periodontology, Indianápolis, v. 6, n. 1, p.23-29, 1995.

JIN, L. J. et al. Global oral health inequalities: task group-periodontal disease. Advances in Dental Research, Washington, v. 23, n. 2, p. 221-6, 2011.

LOPEZ R, Fernandez O, Baelum V. Social gradientes in periodontal disease among adolescents Community Dent Oral Epidemiol 2006.

MICHALOWICZ BS, Hodges JS, Novak MJ, Buchanan W, DiAngelis AJ, Papapanou PN et al. Change in periodontitis during pregnancy and the risk of pre-term birth and low birthweight. J Clin Periodontol. 2009; 36: 308-314.

SADDKI N, Bachok N, Hussain NH, Zainudin SL, Sosroseno W. The association between maternal periodontitis and low birth weight infants among Malay women. Community Dent Oral Epidemiol 2008; 36(4):296-304

SEYMOUR, G. J. et al. Relationship between periodontal infections and systemic disease. Clinical Microbiology and Infection, Oxford, v. 13, n. 4, p. 3-10, 2007.

VAN DYKE, T. E.; VAN WINKELHOFF, A. J. Infection and inflammatory mechanisms. Journal of Periodontology, Indianapolis, v. 40, suppl. 14, p. 1-7, 2013 